\title{
Das kollektive Ritual als Performance? Die Herstellung von religiöser ,Authentizität" in der differenzierten Gesellschaft
}

\author{
Insa Pruisken $(\mathbb{D} \cdot$ Josefa Loebell
}

Eingegangen: 20. April 2021 / Überarbeitet: 13. September 2021 / Angenommen: 21. September 2021 / Online publiziert: 18. Oktober 2021

(C) Der/die Autor(en) 2021

Zusammenfassung Der Beitrag greift Jeffrey Alexanders (2004) These auf, dass die einzelnen Komponenten kollektiver Rituale in der modernen Gesellschaft zwar zunehmend differenziert und somit religiös ,entleert“ werden, in der Inszenierung aber „,re-fusioniert“ werden können. Das Religiöse wird somit subjektiviert, mediatisiert und eventisiert. Rationalisierungsprozesse wie Organisations- und Marktbildung sowie Digitalisierung treiben diese Entwicklung voran. Im empirischen Teil vergleichen wir drei kollektive Rituale aus dem amerikanischen Evangelikalismus, die unterschiedliche Grade der Differenzierung aufweisen: Im Fall der (1) traditionellen Gemeinde wird religiöse Authentizität aus den engen sozialen Beziehungen innerhalb der Gemeinde und zur Denomination erzeugt. (2) Im Megakirchengottesdienst der Lakewood Church wird Authentizität in der professionellen EventInszenierung hergestellt. (3) Die evangelikale Vloggerin hingegen erzeugt Authentizität - und damit eine Bindung an ihr Publikum -, indem Skript und Darstellerin in der Inszenierung vollständig verschmelzen.

Schlüsselwörter Inszenierung · Ritual · Evangelikalismus · Populäre Religion · Mediatisierung $\cdot$ Eventisierung

Insa Pruisken $(\bowtie) \cdot$ Josefa Loebell

Universität Bamberg, Bamberg, Deutschland

E-Mail: insa.pruisken@uni-bamberg.de

Josefa Loebell

E-Mail: josefa.loebell@uni-bamberg.de 


\title{
The collective ritual as Performance? The making of religious "Authenticity" in the differentiated society
}

\begin{abstract}
The contribution takes up Jeffrey Alexander's (2004) idea that although the components of collective rituals become increasingly differentiated in modern society and thus religiously "empty", they can be "re-fused" in the performance. Consequently, religion is subjectivized, mediatized and eventized. Rationalization processes such as the formation of organizations and markets as well as digitalization further this development. In the empirical section, we compare three collective rituals found in American evangelicalism that demonstrate varying degrees of differentiation. In the case of a (1) traditional congregation, religious authenticity is generated from the close social ties within the congregation and to the denomination. (2) In Lakewood Church's megachurch service, authenticity is created in the professionally staged performance. (3) In contrast, the evangelical vlogger creates authenticity and a bond with her audience mainly by fusing completely with the script.
\end{abstract}

Keywords Social Performance $\cdot$ Ritual $\cdot$ Evangelicalism $\cdot$ Popular religion · Mediatization · Eventization

\section{Einleitung}

Der Begriff der ,populären“ Religion ist oft eng verbunden mit der Unterscheidung zwischen einer institutionalisierten Religion, die sich durch Dogmen, Gottesdienste und professionellen Klerus auszeichnet, sowie einer popularisierten, ,volkstümlichen" Variante dieser institutionalisierten Religion, die von Laien dominiert ist und sich durch Magie und Aberglaube auszeichnet (Possamai 2015; Berlinerblau 2001, S. 350). Diese Spannung zwischen institutionalisierter und populärer Religion reflektiert meist eine stratifizierte Gesellschaftsform, in der sich die Mitglieder der oberen privilegierten Klassen mit (unter anderem) religiösen Mitteln von den unteren, weniger privilegierten Klassen abgrenzen (Gramsci 1985; Bourdieu 1993).

Mit dem Aufstieg der Marktgesellschaft und einer sich ausbreitenden Massenkultur löst sich auch die klare Zuordnung von institutionalisierter Religion und höheren Schichten einerseits sowie populärer Religion und unteren Schichten andererseits. In vielen Gesellschaften hat sich das Verhältnis zwischen Religion, Persönlichkeit und Sozialstruktur grundlegend verändert (Kern und Pruisken 2018; Luckmann 1988). Die Produktion von Musik und anderen Kulturgütern wurde im 20. Jahrhundert zunehmend kommerzialisiert und zentralisiert („Kulturindustrie“). Damit verbunden war die Homogenisierung des kulturellen Geschmacks (DiMaggio 1987), die auch die Religion stark beeinflusste (Moore 1995). Für Knoblauch ist daher die Ausbreitung des populären (religiösen) Wissens eng mit einer zunehmenden Auflösung der Grenzen zwischen „Orthodoxie“ und „Heterodoxie“ verbunden (Knoblauch 2018).

Mit der Pluralisierung des religiösen Feldes wachsen in den meisten westlichen Gesellschaften zudem die Spielräume für den Einzelnen, weil die teilweise hochspezialisierten Wissensbestände aus verschiedenen sozialen Wertsphären auf unter- 
schiedliche Weise miteinander verbunden werden können (Simmel 1908, S. 26). In diesem Prozess wird das spezialisierte Wissen subjektiviert (Knoblauch 2002). Gerade mit Blick auf die Religion wird der Übersetzungsprozess in das Subjektive aber immer anspruchsvoller, weil die kulturelle Distanz zwischen dem individuell und kollektiv geteilten religiösen Hintergrundwissen größer wird (Alexander 2004). Innerhalb der institutionalisierten Religion gelingt es z. B. oft nicht, subjektive Erfahrungen der Selbsttranszendenz narrativ zu rahmen. Viele Religionssoziologen sehen in der Konsequenz eine fortschreitende Säkularisierung der Gesellschaft. Beiträge, die sich mit der Popularisierung, ,,Mediatisierung“ oder „Eventisierung“ von Religion beschäftigen, orientieren sich hingegen an Luckmanns These der ,unsichtbaren Religion“. Demnach verschwinde das Religiöse nicht, es verändere nur seine Form (Hjarvard 2013; Hepp et al. 2009; Knoblauch 2000b; Gebhardt et al. 2000; Luckmann 1967). Im Zentrum steht in diesen Theorien die Subjektivierung der Religion.

Der Beitrag greift diese Perspektive auf, rückt aber die Entstehung räumlicher, zeitlicher und kultureller Distanzen durch Rationalisierungsprozesse wie Organisations- und Marktbildung sowie Digitalisierung in den Fokus. Wir beziehen uns auf Jeffrey Alexanders performativen Ansatz (Kern 2017; Alexander 2004), mit dem sich zeigen lässt, wie die zunehmend differenzierten (und dadurch distanzierten) Komponenten religiöser Rituale - kultureller Hintergrund, Skript, Darsteller und Publikum - durch erfolgreiche Inszenierung ,re-fusioniert“ werden. In der Studie werden drei Inszenierungen aus dem evangelikalen Spektrum miteinander verglichen: der Gottesdienst einer traditionellen Gemeinde, der Gottesdienst einer Megakirche sowie der „Vlog“ einer evangelikalen Influencerin. Alle drei Veranstaltungen sind in den USA lokalisiert, unterscheiden sich dabei aber im Grad ihrer räumlichen, zeitlichen und kulturellen Differenzierung. Ziel des Artikels ist es zu zeigen, wie sich mit zunehmender Reichweite der Kommunikation auch die Spielräume für Inszenierungspraktiken vergrößern.

\section{Popularisierung, Subjektivierung und Eventisierung der Religion}

In der Soziologie war die Debatte über die Zukunft der Religion stets an eine gesellschaftstheoretische Perspektive geknüpft: Während die Säkularisierungstheoretiker von einem wahrscheinlichen Zusammenhang zwischen Modernisierung und Säkularisierung ausgehen, sprechen die Kritikerinnen mitunter von einer „Desäkularisierung“ (Berger 2005) oder „Wiederkehr der Götter“ (Graf 2007). Im deutschsprachigen Diskurs war es insbesondere die Wissenssoziologie, die Luckmanns These der „Unsichtbaren Religion“ weiter ausarbeitete und zeigen konnte, dass die Religion nicht aus der Gesellschaft verschwindet, sondern ihre soziale Form ändert (Knoblauch 2009, 2020; Luckmann 1967). Während die institutionalisierte Religion - in Deutschland insbesondere die beiden großen Volkskirchen - an Einfluss verliere, verlagerten sich religiöse Erfahrungen zunehmend in den Bereich der Populärkultur. Das Ergebnis sei ein „Entdifferenzierungsprozess [...] zwischen weltlicher Kultur und sakraler Religion" (Knoblauch 2002, S. 296), in dessen Zentrum die Subjektivierung religiöser bzw. spiritueller Erfahrungen stehe. 
Entdifferenzierung heißt in diesem Fall, dass die als „,hochgradig kompartmentalisiert" empfundene funktional differenzierte Gesellschaft in der subjektiven spirituellen Erfahrung integriert wird: „Ganzheitlichkeit lässt sich also vor dem strukturellen Hintergrund der institutionellen Differenzierung verstehen, die sie voraussetzt und auf die sie (keinesfalls ausdrücklich) Bezug nimmt“ (Knoblauch 2009, S. 127). Beispielsweise sind in den Bereichen der Gesundheit, Bildung und Pflege immer neue Berufe und Rollen entstanden (Gerhards 2001), die den kulturellen Rahmen produzieren und verbreiten, vor dessen Hintergrund ganzheitliche spirituelle Transzendenzerfahrungen überhaupt erst möglich werden. „Die institutionell getrennten, auf unterschiedlichen Legitimitäten basierenden Formen des Wissens werden auf eine Weise vermischt, in der die Unterschiede zwischen religiösem und ökologischem, magischem und medizinischen, alltäglichen und professionellem Wissen verschwinden“" (Knoblauch 2002, S. 303). Die Entdifferenzierung des Wissens ist dabei eng an eine Subjektivierung geknüpft, die mit der klassischen Trennung zwischen religiösen „Virtuosen“ und „Laien“ bricht: „Nicht mehr das, was die Theologie oder das Amt verkündet, wird angenommen, sondern nurmehr das, was man selbst erfahren hat" (Knoblauch 2002, S. 302).

Aus dieser Perspektive ist als logische Konsequenz funktionaler Differenzierung nicht nur Säkularisierung - das heißt: ein Verschwinden der Religion - denkbar, sondern ebenso eine Verschiebung des Religiösen ins Private. In der Folge entstehen unter anderem mediatisierte und eventisierte Formen der religiösen Vergemeinschaftung. Einerseits wird religiöse Kommunikation zunehmend medial vermittelt, andererseits übernehmen die Medien - Film, Musik, Unterhaltung, etc. - religiöse Funktionen (Hjarvard 2013). In der deutschsprachigen Soziologie wurde vor allem die „Eventisierung“ des Religiösen herausgearbeitet (Gebhardt 2018, 2015, S. 416, 2000, S. 28; Pfadenhauer 2008, S. 130; Haken und Wetzels 2017). Während traditionelle Formen religiöser Vergemeinschaftung demnach an Attraktivität verlieren, bieten religiöse Events Möglichkeiten der situativen Vergemeinschaftung in der individualisierten Gesellschaft (Gebhardt 2000, S. 28, 2018, S. 593). Privatisierung und die Mediatisierung bzw. Eventisierung der Religion hängen zusammen, weil medial vermittelte und eventisierte Angebote die Subjektivierung der Religion eher ermöglichen.

Eventisierte Feste, die sich durch ein heterogenes Publikum, Spielräume für subjektive Freiheit in der Partizipation sowie Kommerzialisierungstendenzen auszeichnen, fokussieren ,,identifikationsfähige Inhalte“ und generieren dadurch situativ identitätsstiftende Erlebnisse und Gefühle der Zusammengehörigkeit unter den Teilnehmerinnen (Gebhardt 2000, 2015, 2018). Diverse Studien thematisieren vor diesem Hintergrund Großveranstaltungen wie den Weltjugendtag oder die Kirchentage (Ebertz 2000; Pfadenhauer 2008; Forschungskonsortium WJT 2007). So wird der Weltjugendtag als Versuch einer „katholischen Reterritorialisierung eines sich auflösenden - entgrenzenden und entdifferenzierenden - religiösen Felds“ (Ebertz 2000, S. 358) bzw. als Gelegenheitsstruktur für die Entstehung individueller, situativer Zugehörigkeitserfahrung und Vergemeinschaftung verstanden. Gläubige wollen demnach Religion ,authentisch“" erfahren, ,nicht-authentisches“ Handeln kirchlicher Funktionäre werde hingegen abgelehnt (Forschungskonsortium WJT 2007, S. 15, 42-43). 
Events ermöglichen insofern die Erzeugung von Vergemeinschaftungserfahrungen in einer zunehmend differenzierten Gesellschaft. Anders als bei Ritualen in „einfachen“ Gesellschaften wird die strategische Dimension bei der Inszenierung von Events immer wichtiger (Knoblauch 2000a). Die strategische Dimension ist dabei nicht nur ein zentrales Element von Events. Vielmehr entwickeln wir die These, dass organisierte, medial vermittelte und digitalisierte religiöse Angebote zunehmend performativ inszeniert werden. Wir unterscheiden deshalb zwischen Formen der religiösen Inszenierung im Spannungsfeld von Vergemeinschaftung und Vergesellschaftung und fragen, wie sich kollektive Rituale durch Rationalisierungsprozesse wie Organisations- und Marktbildung sowie Digitalisierung innerhalb des religiösen Feldes verändern. Die Frage, die sich stellt, lautet: Wie verändern sich Rituale im Zuge sozialer Differenzierung?

\section{Kollektive Rituale in traditionellen und modernen Gesellschaften}

Die Analyse von Ritualen in der anthropologischen Forschung basierte zunächst auf der Beobachtung „einfacher“ Gesellschaftsformen. Insbesondere Victor Turner hat in seinen Studien die rituelle Gemeinschaftserfahrung betont, die im Rahmen des ,liminalen Schwellenzustands“ in der „Beseitigung von Sozialstruktur“ mündet (Turner 2005, S. 169). Um zwischen Ritualen in einfachen und differenzierten Gesellschaften zu unterscheiden, führt Turner das Begriffspaar ,liminal“ versus „liminoid“ ein (Turner 1974). Wird der Übergang von einer sozialen Position zur anderen - etwa vom Jugendlichen zum Erwachsenen - in einfacheren Gesellschaften oft durch das Ritual vorgegeben, so ist die Teilnahme an ,liminoiden“ Ritualen wie etwa Rockkonzerten oder Massendemonstrationen in modernen Gesellschaften nicht nur freiwillig, sie steht auch für ein Überschreiten von und den Ausbruch aus gesellschaftlich vorgegebenen Verhältnissen. In modernen - kulturell fragmentierten und sozial differenzierten - Gesellschaften werden liminale Rituale daher zunehmend liminoid und somit zu „Performances“, deren Gelingen davon abhängt, dass sie von den beteiligten Akteuren als wahrhaftig und authentisch erlebt werden (Alexander 2004).

Alexander (2004) arbeitet den Zusammenhang zwischen fortschreitender gesellschaftlicher Differenzierung und dem Wandel von Ritualen überzeugend heraus. Grundgedanke seiner Theorie ist die Vorstellung, dass die Komponenten, aus denen ein Ritual besteht, in einfacheren Gesellschaften ,fusioniert“ sind, wohingegen sie sich in differenzierteren Gesellschaften immer stärker auseinanderentwickeln. In traditionellen Gesellschaften werden Rituale durch vorherrschende Statusordnungen und Moralvorstellungen strukturiert (Turner 2005): Sie verkörpern die Sozialstruktur. In modernen Gesellschaften werden Sozialstruktur, Persönlichkeit und Kultur hingegen immer unabhängiger voneinander (Luckmann 1988), so dass religiöse Weltansicht, persönlicher Geschmack und soziale Position sich nicht mehr zwangsläufig überlagern. Unter dieser Bedingung scheitern kollektive Rituale oft, weil es nicht gelingt, die verschiedenen Interessen und Bedürfnislagen eines fragmentierten Publikums zu überbrücken. In modernen Gesellschaften werden Rituale deshalb zunehmend als „Events“ von Organisationen inszeniert. Sie leisten so die 
Vermittlungsarbeit zwischen individuellen Erfahrungen und gesellschaftlichen Erwartungen.

Die Authentizität eines Rituals muss dabei jedes Mal neu im Rahmen einer Inszenierung (Performance) erzeugt werden, ohne das Publikum bemerken zu lassen, dass es sich um eine Inszenierung handelt (Alexander 2004; Fischer-Lichte und Pflug 2000; von Appen 2013; Kreuzer 2011; Funk und Krämer 2011; Hans 2017). Im Anschluss an Taylor lässt sich „Authentizität“ als Treue des Einzelnen zu sich selbst bzw. zur eigenen Natur definieren (Taylor 2003, S. 55). Authentizität stellt das eigentliche, echte und restlose Aufgehen in der eigenen Rolle dar und ist klassischerweise als Gegenbegriff zur Entfremdung (Henning 2015, S. 24) oder „Rollendistanz“ (Goffman 1973) zu verstehen. Dementsprechend verschiebt sich der Fokus auf die strategische Inszenierung von Authentizität im Sinne einer glaubhaften Darstellung des Nicht-Darstellens (Reichertz 2001, S. 24). Je stärker die einzelnen Komponenten eines kollektiven Rituals voneinander differenziert sind, desto mehr Aufwand müssen die beteiligten Akteure betreiben, um sie miteinander zu einem authentischen Ganzen zu verschmelzen. Kann das Publikum den Ablauf des Rituals und die subjektive Erfahrung der Teilnehmerinnen (Darsteller) nicht nachvollziehen, miterleben und verstehen, drohen Events zu scheitern (Knoblauch 2000a, S. 42-43). In den Mittelpunkt rückt die „Erfahrung von etwas Wirklichem“ (Knoblauch 2000a, S. 43).

Die differenzierten Elemente eines kollektiven Rituals müssen in modernen Gesellschaften somit immer wieder neu ,re-fusioniert“ werden, indem das aufgeführte „Skript“ (siehe unten) in zwei Richtungen erst getrennt und dann wieder angebunden wird: mit dem kulturellen Hintergrundwissen des Skripts in der einen Richtung und dem Publikum in der anderen Richtung (Alexander 2004, S. 551). „The aim is to create, via skillful and affecting performance, the emotional connection of audience with actor and text and thereby to create the conditions for projecting cultural meaning from performance to audience" (Alexander 2004, S. 547). Alexander (2004, S. 529-533) zufolge bestehen Rituale bzw. Performances aus sechs Komponenten, die in Abhängigkeit von der Sozialstruktur schwach oder stark voneinander differenziert sein können:

(1) Das gemeinsame kulturelle Hintergrundwissen (,Systeme der kollektiven Repräsentation") beinhaltet auf einer allgemeinen Ebene die existenziellen, emotionalen und moralischen Bezüge der Inszenierung. Beispielsweise bildet im christlichen Gottesdienst die jeweilige Konfession oder Denomination den kollektiven Rahmen des Rituals. Das durch die religiöse Zugehörigkeit geteilte religiöse Hintergrundwissen konstituiert die Basis der Gemeinschaft. Für Alexander ist dieser symbolische Bezugsrahmen durch einen Vordergrund und einen Hintergrund strukturiert. Während der Hintergrund durch Glaubensgrundsätze, Vorstellungen und biblisches Wissen konstituiert wird, enthält der Vordergrund die verhaltensrelevanten Narrative und Skripts, die im Ritual aufgeführt werden. Das Skript - sozusagen das „Drehbuch“ verweist auf den kollektiven Hintergrund, ähnlich wie das Kreuz am Altar auf die Leidens- und Erlösungsgeschichte Jesu. Es bildet den „Referenztext“, an dem sich die Akteure während der Inszenierung orientieren (Alexander 2004, S. 530). Je nach christlicher Tradition ist das Skript eines Gottesdienstes durch die Liturgie und die Agende unterschiedlich strukturiert. Im Zuge der Popularisierung des Religiösen werden die von der jeweiligen kirchlichen Orthodoxie bewahrten Wissensbestände 
und Symbolsysteme jedoch immer stärker mit ,popkulturellen“ Wissensbeständen vermischt oder ,,verwässert“. Der Prozess der Entgrenzung (Knoblauch 2002) von populärer Kultur und Religion beinhaltet somit zunächst eine Differenzierung zwischen Hintergrundkultur und Skript: Erst wenn die Akteure sich von ihrem starken kulturellen Hintergrund lösen, können sie sich dem Populären zuwenden.

Das Skript muss durch (2) Darstellerinnen - Schauspieler im Theater, Pastorinnen und Priester in religiösen Ritualen - für das Publikum gespielt, gesprochen und in Szene gesetzt werden.

Das (3) Publikum besteht zunächst aus den Personen, die auf die Partizipationsangebote einer Organisation zugreifen. Welche Personen das religiöse Publikum konstituieren, hängt von den Mitgliedschaftsregeln ab, die die Teilnahme an kollektiven Ritualen regeln.

Für die Darstellung des Skripts benötigen die Akteure stets bestimmte (4) Mittel der symbolischen Reproduktion. Physische Objekte wie Bühnenelemente, Requisiten oder Kostüme ,serve as iconic representations to help them dramatize and make vivid the invisible motives and morals they are trying to represent" (Alexander 2004, S. 532).

Mit der zunehmenden Ausdifferenzierung der Komponenten eines kollektiven Rituals gewinnt die (5) Inszenierung des Skripts an Autonomie: Das Skript wird unabhängiger von der kulturell geteilten Vorstellung, wie das Ritual traditionell abzulaufen hat. In der katholischen Kirche ist es beispielsweise die Liturgie, die das Skript definiert. Kollektiver Hintergrund und Skript sind in diesem Fall eng aneinandergekoppelt. Lösen sich kollektiv geteilter Hintergrund und Skript voneinander, setzt dies oft auch die Ausdifferenzierung einer professionalisierten, organisatorischen Rollenstruktur - Regie, Dramaturgie, Maske, Bühnenbild, Musik, Licht etc. in Gang. Im Mittelpunkt stehen dann die Planung und gezielte Erzeugung von religiösen Emotionen. Dies gilt aber nicht nur für religiöse Rituale: Auch politische Events oder Protestkundgebungen folgen oft einer choreografierten Inszenierung mit Spannungsbogen und -steigerung.

Die Performance ist darüber hinaus stets durch spezifische (6) soziale Machtverhältnisse strukturiert. Alexander deutet hier an, dass kollektive Rituale unter dem Einfluss von institutionellen Rahmenbedingungen stehen, die festlegen und kontrollieren, wer teilnehmen, sprechen oder auftreten darf bzw. muss. Die Frage, wie institutionelle, strategische und organisatorische Bedingungen die Performance beeinflussen, greifen wir in dieser Studie auf und entwickeln sie weiter.

\section{Organisationsbildung, Vermarktlichung und Digitalisierung}

Welche sozialen und organisatorischen Rationalisierungsprozesse treiben die Differenzierung von Ritualen in der modernen Gesellschaft an? Der Wandel des religiösen Feldes hat insbesondere in den USA eine Vielzahl neuer institutioneller und organisatorischer „Sozialformen“ (Heiser et al. 2014; Krech et al. 2013; Krüggeler et al. 1999) hervorgebracht. Innerhalb dieser Sozialformen werden Machtverhältnisse auf unterschiedliche Weise geregelt. Zudem unterscheiden sie sich durch den Grad der sozialen Differenzierung (Geser 1999; Petzke 2013). Die kommunikative Reichweite 
formaler Organisationen ist typischerweise höher als die einer lokalen Gemeinschaft. Events werden daher meist von Organisationen professionell geplant und durchgeführt. Events sind somit nicht nur technisch voraussetzungsvoll, sondern gehen mit einem Bedeutungswachstum von formalen Organisationen einher (Luhmann 1972a). Dies lässt sich am Fall des Evangelikalismus in den USA anschaulich illustrieren, wo Marktbildung und Digitalisierung schon wesentlich stärker vorangeschritten sind als in vielen, insbesondere europäischen Ländern.

In den USA entstanden im 19. Jahrhundert die sogenannten Denominationen als wohl erste Form einer religiösen Mitgliedsorganisation jenseits des europäischen Staatskirchenmodells (Mead 1954; Niebuhr 1957; Ammerman 2016). Dabei waren es vor allem die Methodisten und Baptisten, die sich mit Hilfe hierarchisch-zentralisierter Organisationen koordinierten (Balik 2014, S. 5). Die Gläubigen konnten relativ frei wählen, welcher Denomination sie sich anschließen wollten. Diese Struktur breitete sich über das gesamte besiedelte Gebiet der USA aus. Die primäre religiöse Identifikation verschob sich zugleich von lokal gebundenen Gemeinden auf theologisch, ethnisch und kulturell (relativ) homogene nationale Denominationen.

Obwohl diese neue - zunächst hauptsächlich protestantische - Organisationsform im Prinzip religiösen Wettbewerb zuließ und den Gläubigen neuartige Wahlmöglichkeiten eröffnete, blieb die einzelne Gemeinde fest an die kulturelle Hintergrundstruktur der Denomination gebunden. Die neuen Denominationen verlangten zwar von ihren Mitgliedern ein authentisches commitment für den Glauben. Davon zeugten etwa die großen Erweckungsversammlungen (als religiöse „Events“), zu denen die Besucherinnen teilweise von weither anreisten, um massenhaft an spektakulären Glaubenserfahrungen teilzuhaben (Moore 1995). Die Denominationen sind folglich bereits Organisationen, die erfolgreich religiöse Events inszenieren, ihre Mitglieder bleiben aber oft eng gebunden an ethnische, regionale, lokale und politische Zugehörigkeiten (Niebuhr 1957). Die Denomination blieb insofern für lange Zeit ein zentraler Knotenpunkt in einem dichten Netzwerk lokaler Beziehungen, in dem verschiedene Gruppenzugehörigkeiten zusammenliefen (Kern und Pruisken 2020). Die einzelne Gemeinde ist in dieser Konstellation aber nur eine Subeinheit der Denomination und hat in vielen Denominationen nur wenig Spielraum etwa bei der Inszenierung der Gottesdienste. Zumeist ist sie in ihrer Identität und bei der Durchführung von Ritualen stark von der Tradition der Denomination geprägt.

Seit den 1970er-Jahren hat sich diese Ausgangskonstellation innerhalb des protestantischen Feldes jedoch verändert: Die Zugehörigkeit zu einer Denomination ist für das Alltagsleben immer weniger relevant. Dies zeigt sich etwa an einer wachsenden religiösen Mobilität und Wechselbereitschaft zwischen Gemeinden und Denominationen (Suh und Russell 2015). ${ }^{1}$ Dieser Wandel ging einher mit dem ökonomischen Aufstieg einer breiten Bevölkerung und einer damit verbundenen Nivellierung kultureller Differenzen zwischen bislang getrennten regionalen, sozialen und ethnischen Gruppenzugehörigkeiten (Kern und Pruisken 2018; Herberg 1960). Als treibende Kräfte dieser Entwicklung gelten bis heute die relative Angleichung des Bildungs-

\footnotetext{
1 Dazu passt, dass auch innerhalb des protestantischen Feldes der Anteil der Gemeinden wächst, die sich mit keiner Denomination identifizieren: Zwischen 1998 und 2012 erhöhte er sich von $18 \%$ auf $25 \%$. Ihr Anteil an den Gläubigen stieg parallel von $14 \%$ auf $21 \%$ (Chaves et al. 2014).
} 
niveaus, der Massen- und Medienkonsum sowie die hohe regionale Mobilität (Herberg 1960, S. 112). Die enge Verbindung zwischen der Denomination und anderen Gruppenzugehörigkeiten hat sich dadurch deutlich gelockert.

Zudem breitete sich ein neuer Gemeindetyp aus - die sogenannte Megakirche -, die sich im Sinne einer religiösen Marktlogik immer weniger am Kirchenverständnis des Denominationalismus orientiert (Kern und Schimank 2013). ${ }^{2}$ Megakirchen verstehen sich nicht mehr als Subeinheit ihrer Denomination, die auf die klassischen religiösen „Funktionen“ (Warner 1994, S. 63) spezialisiert ist: Gottesdienst, Erziehung, Mission, Diakonie, Gemeinschaftsbildung etc. Vielmehr handelt es sich um relativ autonome „organisatorische Akteure“ (Krücken und Meier 2006), die sich vergleichsweise unabhängig von den Leitwerten und dem Expertenwissen der Denomination eigenständig Ziele setzen und dabei zumeist eine konsequente Wachstumsstrategie verfolgen.

Damit wird ein Prozess in Gang gesetzt, in dem lokale Gemeinden sich zunehmend als religiöse Anbieter verstehen, die einem Publikum aus religiösen Konsumenten gegenüberstehen, um dessen Aufmerksamkeit sie mit anderen Gemeinden konkurrieren (Weber 1980, S. 117; vgl. auch Miller 2013; Moberg und Martikainen 2018; Sanders 2015; Sandıkcı 2018). Die Akteure kalkulieren dabei ihr Handeln darüber hinaus zunehmend strategisch unter rationalen Gesichtspunkten (Callon und Muniesa 2005). Aus der generalisierten indirekten Reziprozität ${ }^{3}$ der religiösen Gemeinschaft wird eine marktorientierte Tauschbeziehung: Die Gemeinden bieten ihren Gläubigen religiöse Heilsgüter (Gottesdienste, Seelsorge, Bibelschule etc.) und erhalten im Gegenzug deren commitment (Engagement). Wächst eine Gemeinde, so bedeutet dies, dass auch ihre Möglichkeiten wachsen, das Engagement der Gläubigen gezielt für vielfältige religiöse Zwecke einzusetzen. Megakirchen konzentrieren mittlerweile große Mengen der Ressourcen, die im religiösen Markt aufgebracht werden (Chaves 2006), und sind daher auch in der Lage, in die Inszenierung aufwändiger religiöser Events zu investieren. Sie steigern insofern ihre Machtgrundlage und können beeinflussen, wer auftritt, welche Themen adressiert werden und wer am Gottesdienst teilnehmen darf. Die meisten Megakirchen lösen sich so vom kulturellen Hintergrund der Denomination ab und werden auf der Organisationsebene zu autonomen Akteuren, so dass Skript und kultureller Hintergrund des Rituals bzw. der Performance auseinanderdriften.

\footnotetext{
${ }^{2}$ Die Frage, inwiefern die Religion ein Markt ist oder Vermarktlichungsprozessen unterliegt, ist Kernbestand religionssoziologischer Debatten, vergleiche nur Berger (1963); Iannaccone (1992); Finke und Stark (1988). Wir orientieren uns in diesem Beitrag an einem soziologischen Verständnis des Martkbegriffs, wie es in der neueren Wirtschaftssoziologie entwickelt wurde, siehe Beckert (2009); Fligstein und Dauter (2007). Für eine soziologische Perspektive auf religiöse Märkte siehe Kern und Pruisken (2018); Pruisken et al. (2021).

${ }^{3}$ Wir beziehen uns hier auf die Unterscheidung zwischen indirekter, generalisierter Reziprozität und direkter Reziprozität, siehe Molm et al. (2007). Im ersten Fall gibt Akteur A ein Gut an Akteur B und erhält dafür ein Gut zurück, allerdings nicht zwangsläufig von Akteur B selbst, sondern von irgendeinem Akteur aus der Gemeinschaft. Im Fall der direkten Reziprozität - die dem Markttausch näherkommt - treten die Akteure A und B hingegen in eine direkte Tauschbeziehung. Der Akteur A erwartet nun eine direkte Gegenleistung für sein gegebenes Gut, vgl. zu dieser Diskussion in der Religionssoziologie Pace (2006); Stolz (2006).
} 
Mit der Ausbreitung digitaler Plattformorganisationen werden zunehmend ,evaluative Infrastrukturen“ (Kornberger et al. 2017) bereitgestellt, die personale und organisationale (religiöse) Akteure nicht nur sozial miteinander vernetzen, sondern Objekte und Personen kulturell kategorisieren, quantifizieren, standardisieren und evaluieren. Auf diese Weise generieren Plattformen neue Formen der religiösen Partizipation. Gemeinden und Religiöse Influencer streamen ihre Videos live im Netz und lassen ihre Follower an ihrem (Gemeinde-)Leben teilnehmen. Gleichzeitig strukturieren sie diese Vernetzungs- und Evaluationsprozesse, indem sie die technischen und kulturellen Voraussetzungen schaffen, die den Austausch zwischen religiösem Inhalt (als „Ware“) und commitment ermöglichen (van Dijck 2013, S. 145). Während commitment auf der Gemeindeebene noch bedeutet, dass man den Gottesdienst besucht, an Gemeindeaktivitäten teilnimmt oder Geld spendet, bedeutet es auf der Plattformebene, dass man Inhalte generiert, ansieht, teilt, kommentiert, ,liked“ oder bewertet. Je mehr die Plattformen miteinander um die Aufmerksamkeit der Nutzer konkurrieren (Gillespie 2010, S. 351; Hracs und Webster 2020, S. 2), desto vielfältiger werden für die individuellen Gläubigen die Möglichkeiten, sich mehr oder weniger aktiv einzubringen.

Insofern stellen Plattformen eine komplette Infrastruktur zur Verfügung, die es erlaubt, (religiöse) Performances zu inszenieren und einer breiten Öffentlichkeit zu Verfügung zu stellen (Ostertag 2021, S. 115). Die Bindungen zwischen Publikum und Anbieter werden auf diese Weise allerdings raumzeitlich entgrenzt und damit auch fragmentiert (Einspänner-Pflock und Reichmann 2014, S. 58). Die Möglichkeiten der Personalisierung der für die Nutzer sichtbaren Inhalte steigen durch die Plattformalgorithmen (van Dijck 2013, S. 149). Das Publikum wird so unabhängiger von den Anbietern und kann relativ unabhängig von räumlichen und zeitlichen Begrenzungen zwischen unterschiedlichen Angeboten wählen.

\section{Fallvergleich: Der Prozess der Fusionierung}

Der Fallvergleich orientiert sich an den im Abschnitt 3 entwickelten Kriterien. Wir vergleichen dementsprechend die religiösen Inszenierungen, die im Rahmen von drei verschiedenen Formen der religiösen Vergemeinschaftung produziert werden. Diese unterscheiden sich durch den Grad der sozialen Differenzierung.

(1) Im ersten Fall untersuchen wir eine Live-Stream-Aufnahme eines Gottesdienstes einer kleineren, denominationsgebundenen Gemeinde aus dem Süden der USA. Der Gottesdienst wird von etwa 500 bis 800 Personen wöchentlich besucht. Die Gemeinde gehört der Cooperative Baptist Fellowship an, einer baptistischen Denomination, die sich Anfang der 1990er-Jahre von der Southern Baptist Convention abgespalten hat. Im Gegensatz zur SBC erlaubt die CBF Frauen das Predigen. Die Gemeinde verfügt über für den Baptismus traditionelle demokratische Leitungsstrukturen, bei denen die Mitglieder als Ehrenamtliche in die Leitung der Gemeinde eingebunden werden. Die Gemeinde streamt ihre Gottesdienste meist live im Netz und verfügt deshalb über eine professionelle technische Ausstattung. Dennoch ist der Livestream nur über die Website der Kirche erreichbar und richtet sich dementsprechend hauptsächlich an die Mitglieder und Bekannte der Gemeinde, die nicht 
anwesend sein können. Aus Interviews mit Pastoren und Mitgliedern der Gemeinde $(2013,2020)$ wissen wir, dass die Gemeinde sich als Gemeinschaft versteht und nicht zwischen sich als „Anbietern“ und den Mitgliedern als „Konsumenten“ differenziert.

(2) Den zweiten Fall bildet die Live-Stream-Aufnahme eines Gottesdienstes der Lakewood Church, einer unabhängigen „Megakirche“ mit starker Marktorientierung und Live-Stream-Angeboten in Houston, Texas. Die Lakewood Church ist mit 40.000 regelmäßigen Gottesdienstbesucherinnen pro Woche die größte Megakirche der USA. Seniorpastor Joel Osteen und seine gesamte Familie sind in den USA öffentliche Personen. Die Gottesdienste - „worship experiences“4 - werden regelmäßig live im Fernsehen übertragen. Die Osteens leben vor allem vom Verkauf ihrer Bücher, die oft New York Times Bestseller waren. ${ }^{5}$ Die Gemeinde selbst hat etwa 350 Mitarbeiter und verfügt daher über eine professionalisierte Rollenstruktur. Die Gemeinde ist eine „501(c)(3)“-Organisation und hat somit den Status der Gemeinnützigkeit.

(3) Den dritten Fall bildet ein über $500.000 \mathrm{Mal}^{6}$ aufgerufenes YouTube Video einer christlichen Social-Media-Influencerin, die in diesem Video ihr Bekehrungserlebnis teilt. ${ }^{7}$ Die Influencerin Kirby Kelly, die sich „Kirby is a Boss“" nennt und in Texas einer evangelikalen Gemeinde angehört, ${ }^{8}$ ist Selbstaussagen zufolge ,,in ministry" und verbreitet über diverse Kanäle christliche Heilsbotschaften, Lifestyle-Tipps oder lässt Followers an ihrem persönlichen Glaubensleben teilhaben. Sie bespielt einen Podcast und ist in sozialen Netzwerken wie Instagram, Twitter, Snapchat und TikTok aktiv. Im Mittelpunkt ihres Wirkens steht jedoch die Multimedia-Plattform YouTube. ${ }^{9}$ Dort lädt Kelly seit 2012 Videos hoch, in denen sie über ihren Glauben, christliche Lebensführung und Lebensführungs-Tipps spricht oder ihren Alltag im Vlog-Format präsentiert.

Zur Interpretation der Inszenierungen beziehen wir uns, neben einer systematischen Analyse der drei Videos entlang der Elemente einer Performance, auf weitere Daten: (1) Im Falle des Gottesdienstes von Fall 1 auf 15 ein- bis zweistündige Interviews, die wir vor Ort mit Pastoren und Gemeindemitgliedern geführt haben, sowie auf Beobachtungsprotokolle eines Gottesdienstbesuches und einer Kirchenführung vor Ort in den Jahren 2013 und 2020. Zudem haben wir für diese Gemeinde fünf weitere gestreamte Gottesdienste dokumentiert. (2) Im Falle des Gottesdienstes der Lakewood Church haben wir ebenfalls 2013 einen Gottesdienst besucht und seitdem regelmäßig gestreamte Live-Gottesdienste dokumentiert (insgesamt zehn). (3) Im Falle der Influencerin Kirby Kelly haben wir über einige Wochen hinweg im Februar und März 2021 ihre aktuellen und vergangenen Online-Auftritte und -Aktivitäten verfolgt, protokolliert und ausgewertet.

\footnotetext{
${ }^{4}$ https://www.lakewoodchurch.com/lakewood-experience.

5 https://www.lakewoodchurch.com/store/books.

${ }^{6}$ Das Video (https://www.youtube.com/watch?v=_8uStTjovsY) wurde bis zum 26.03.2021 520.633 aufgerufen.

7 https://www.youtube.com/watch?v=_8uStTjovsY.

8 https://www.instagram.com/p/CM3CR9rMDpJ/.

${ }^{9}$ Eine Selbstbeschreibung der Influencerin sowie eine Übersicht über ihre Online-Kanäle lassen sich auf folgender Website einsehen: https://kirby-kelly.com/about-me.
} 
Die Auswertung folgt den Prinzipien der qualitativen Inhaltsanalyse nach Mayring (2015). Die zentralen Kategorien für die Analyse der drei untersuchten Rituale bilden der Ablauf, die Inszenierungstechniken, die Darstellung der religiösen Inhalte sowie die Einbindung des Publikums. Im ersten Schritt der Analyse wurde der Ablauf der Rituale dokumentiert. Da in allen drei Fällen die religiösen Inszenierungen in etwa dem gleichen Ablauf folgen - das macht sie zu einem Ritual - wählen wir für die folgende Analyse jeweils eine Inszenierung aus, die detailliert analysiert wird. Im zweiten Schritt wurden die in Kapitel 2 erarbeiteten theoretischen Kriterien am Material weiterentwickelt. Im Zentrum der Analyse stand dabei die induktive Anreicherung der Kategorien für den hier untersuchten Fall religiöser Rituale. Für jede der drei Inszenierungen wurden zunächst die Mittel der symbolischen Reproduktion, Inszenierungstechniken, Skripte, Inhalte und Publikum beschrieben sowie Ähnlichkeiten und Unterschiede herausgearbeitet. Die drei ausgewählten Videos haben wir dazu in MaxQDA geladen und entsprechende Passagen kodiert. Im dritten Schritt der Analyse wurde untersucht, welche Komponenten des Rituals fusioniert sind bzw. durch die Inszenierung re-fusioniert werden und wie auf diese Weise Authentizität erzeugt wird.

\subsection{Fall 1: Die ,traditionelle“ evangelikale Gemeinde Verhältnis zwischen kulturellem Hintergrundwissen (1) und Darstellerinnen (2)}

Der Gottesdienst der traditionellen Gemeinde ist von baptistischen Konventionen geprägt. Der Baptismus selbst folgt keiner festgeschriebenen Liturgie, weshalb baptistische Gemeinden größere Freiheiten bei der Interpretation des Skriptes als beispielsweise katholische oder episkopale Kirchen haben. Während des Gottesdienstes wird ein etwa achtjähriges Mädchen getauft. Der Ablauf der Taufe orientiert sich an der Tradition, bei der der Täufling vom Pastor im Baptisterium ganz unter Wasser gedrückt, ein Taufspruch gesprochen wird und der Täufling sich in eigenen Worten zu Jesus bekennt. Der Pastor trägt bei der Taufe eine weiße Robe, ebenso wie die Mitglieder des Jugend- wie auch des Kinderchores. Traditionelle Musik mit Orgel, Glocken und Chor wechseln sich mit Predigt und Lesungen ab.

Die Predigt des Pastors - bei der er einen Anzug trägt - reflektiert das etablierte protestantische Motiv des Gläubigen als ,Werkzeug Gottes“, der im Namen der Kirche aktiv handelt und Gutes tut (Weber 1988, S. 538). Der Pastor weicht in seiner Interpretation nur wenig vom Skript des etablierten baptistischen Protestantismus ab. Beispielhaft erläutert er soziale Aktivitäten der Gemeinde in Texas und der Welt, um die Bedeutung christlichen Handelns hervorzuheben. Die professionelle Rolle als predigender Pastor und die soziale Rolle als Leiter der Gemeinde und Familienvater fallen dabei kaum auseinander. Das entscheidende Kriterium ist hier, dass die Mitglieder den Pastor als Familienvater - seine Frau, seine Kinder, sein Haus - tatsächlich persönlich kennen und im Kirchenalltag erleben. Insofern ist auch der Spielraum, den der Pastor für die spielerische und emotionale Ausgestaltung während der Predigt hat, in einer kleinen und dadurch geringer differenzierten Gemeinde wesentlich schwieriger als in einer Megakirche, in der der Pastor normalerweise nicht alle Gemeindemitglieder und Gottesdienstbesucherinnen persönlich kennt. In der untersuchten Gemeinde wird die innere, emotionale Beziehung des 
Akteurs zum Skript auf der Bühne daher nicht aufgeführt. Die subjektive Religiosität des Pastors, seine religiösen Emotionen und Erfahrungen spielen in der Predigt nur eine geringe Rolle. Die Authentizität des Pastors wird von den Gemeindemitgliedern nur wenig in Frage gestellt, weil sie Teil einer Inszenierung der Gemeinde als Gemeinschaft ist, in der die Beziehungen untereinander eng sind. Die familiäre Athmosphäre innerhalb der Gemeinde wurde von Interviewpartnern wiederholt betont. Für das Gelingen authentischer Bindungen spricht, dass es der Gemeinde immerhin seit einigen Jahrzehnten gelingt, eine stabile Gruppe von etwa 800 aktiven Mitgliedern zu halten.

Am Gottesdienst nehmen etwa 300 Besucherinnen teil, die größtenteils formal gekleidet sind und ein relativ hohes Maß an Homogenität hinsichtlich sozialstruktureller Merkmale aufweisen. Während die protestantische oder katholische Volkskirche Mitglieder typischerweise aus einem räumlich abgegrenzten Einzugsgebiet der Gemeinde rekrutiert, konstituiert sich das Publikum im amerikanischen Denominationalismus stärker auf freiwilliger Basis, aber immer noch abhängig von ethnischen, sozialen und kulturellen Segregationslinien (Niebuhr 1957). Entsprechend zeigen sich auch bezüglich des Verhältnisses von kulturellem Hintergrund, Skript und Publikumsstruktur keine starken Differenzierungstendenzen. Das Publikum der betrachteten Gemeinde ist nach wie vor relativ homogen und scheint ähnlich sozialisiert.

\section{Verhältnis von Mitteln der symbolischen Reproduktion (4), Inszenierung (5),} Darstellern (2) und Publikum (3) Der Gottesdienst findet in einem 1930 erbauten - für US-amerikanische Verhältnisse - ,traditionellen“ Kirchengebäude statt, das mit Kirchenbänken aus Holz und roten Samtbezügen sowie bunten Kirchenfenstern ausgestattet ist. Der Kirchenraum ist mit Blumen und Kerzen feierlich geschmückt. Die Gemeinde verfügt zudem über eine professionelle technische Ausstattung. Die Aufzeichnung des Gottesdienstes entspricht dem Niveau der in Deutschland üblichen Fernsehgottesdienste von ARD und ZDF. Auffällig ist die gezielte symbolische Betonung von Gemeinschaftsgefühl. Nach der vollzogenen Taufe des kleinen Mädchens bittet der Pastor erst die Familie des Mädchens aufzustehen, dann alle Personen, die das Mädchen auf dem Weg zur Taufe begleitet haben (in der Sonntagsschule etc.), und zum Schluss die gesamte Gemeinde, von der das Mädchen nun ein Teil ist. Damit wird eine Grenze zwischen der Gemeinde als Gemeinschaft und der Außenwelt gezogen und kollektive Zugehörigkeit markiert.

Gleichzeitig sind die Besucherinnen des Gottesdienstes nicht nur Zuschauer, sondern nehmen teilweise aktiv am Gottesdienst teil. Die Eltern der Kinder der verschiedenen Chöre sitzen im Publikum. Die Familie des getauften Mädchens sitzt ebenfalls im Publikum, so wie einige andere im Gottesdienst Involvierte, die sich zwischendurch wieder unter die Besucher begeben. Bei diesem liminalen Ritual (Taufe!) führt sich die Gemeinde selbst auf. Die Grenze zwischen Publikum und Darstellern ist insofern schon fusioniert, und der Grad der psychologischen Identifikation des Publikums mit dem Ritual ist hoch. Der Gottesdienst richtet sich an diejenigen, die mit der baptistischen Denomination vertraut sind. Die Beziehungen zwischen den Gemeindemitgliedern sind eng. Man kennt sich aus der Sonntagsschule, aus Bibelkursen und anderen gemeinsamen Aktivitäten. Die Gemeinde kann sich 
darauf verlassen, dass es in ihrer lokalen Metropolregion eine ausreichend große Publikumsnische gibt, die sich von ihrem Angebot überzeugen lässt. Die Übertragung des Gottesdienstes im Internet ist zwar professionell, da sie aber lediglich über die Webseite der Gemeinde abgerufen werden kann, richtet sie sich hauptsächlich an diejenigen, die bereits mit der Gemeinde vertraut sind. Die kulturelle Extension der Darstellung bleibt daher relativ beschränkt und partikular. Sie wird nicht auf größere Bevölkerungsgruppen ausgeweitet.

Die Authentizität der im Ritual erzeugten Emotionen entsteht in diesem Fall nicht nur aus der räumlichen Ko-Präsenz, sondern aus bestehenden persönlichen und kulturellen Bindungen. Kultureller Hintergrund, aufführende Akteure und das Publikum sind in geringem Maße differenziert. Gleichzeitig wird an diesem Beispiel auch deutlich, dass die Gemeinde durch ihre stärkere Denominationsbindung nur wenig Spielraum hat, ihren Gottesdienst zu emotionalisieren und zu ,popularisieren“.

\subsection{Fall 2: Der Gottesdienst als Performance: ,Lakewood Church“ Verhältnis zwischen kulturellem Hintergrundwissen (1) und Darstellerinnen (2)}

Die Lakewood Church gehört hingegen keiner Denomination an und ist daher im Hinblick auf die Inszenierung ihres Skriptes weitgehend frei. Den kulturellen Hintergrund bilden der amerikanische Evangelikalismus und das pfingstlerisch geprägte „Prosperity Gospel“ (Bowler 2013): „Osteen's approach to the fulfillment of the self are comparable to similar products on the market that combine self-help, positive thinking, and the pursuit of happiness and self-realization with spirituality" (Rakow 2013, S. 486). Der Spielraum, den die Gemeinde bei der Interpretation der Bibel hat, ist dementsprechend größer als im ersten diskutierten Fall. Osteen ist beispielsweise bekannt für sein selbst erdachtes Glaubensbekenntnis und seinen die Predigt stets einleitenden Bibelbekenntnisspruch:

This is my Bible. I am what it says I am. I can do what it says I can do. Today, I will be taught the Word of God. I boldly confess: My mind is alert, My heart is receptive. I will never be the same. I am about to receive The incorruptible, indestructible, Ever-living seed of the Word of God. I will never be the same. Never, never, never. I will never be the same. In Jesus name. Amen (Joel Osteen, Lakewood Church) ${ }^{10}$.

Das Glaubensbekenntnis und der oben genannte Spruch werden auf großen Flatscreens zum Mitlesen angezeigt, so dass ihn alle Anwesenden gemeinsam sprechen können. Dafür braucht es kein besonderes Hintergrundwissen über den Glauben. Osteen hat somit - im Sinne einer „Markenbildung“ - kognitiv vereinfachte Rituale eingeführt, die durch das gemeinsame Sprechen das Zusammengehörigkeitsgefühl stärken.

Die Distanz zwischen Akteur und Skript bzw. kulturellem Hintergrund wird im vorliegenden Fall dementsprechend größer, weshalb das indexikale Zeigen auf oder das Vorlesen aus der Bibel nicht ausreichen, um die Botschaft zu übermitteln. Die religiöse Botschaft wird deshalb insbesondere mit Hilfe von Musik und Licht emo-

10 https://www.youtube.com/watch?v=6Gqu1 Ai7xNk. 
tional aufgeladen. Osteens Predigt ist immer gleich strukturiert: Er startet mit ,something funny" und erzählt dann eine biblisch gerahmte Anekdote, die den kulturellen Hintergrundrahmen des Prosperity Gospel untermauert. Die Distanz zum Publikum ist vergleichsweise groß, wird aber dadurch überbrückt, dass dem Publikum im Anschluss an den Gottesdienst persönliche Treffen ermöglicht werden. Anders als im ersten Fall kennen die Gottesdienstbesucherinnen den Pastor nicht persönlich. Sie wissen daher auch nicht, wie er beispielweise seine Rolle als Familienvater tatsächlich ausfüllt. Die ,Aura des Heiligen“ wird stattdessen durch eine starke Abgrenzung zwischen charismatischem Pastor und „Fans“ (Roose et al. 2017) räumlich aufrechterhalten.

Dabei konstituiert sich das Publikum nicht allein aus den Mitgliedern, sondern umfasst auch viele ungebundene Besucher. Den Gottesdienst besuchen zum einen viele unregelmäßige Besucherinnen, die z. B. von außerhalb kommen. Zum anderen wird der Gottesdienst im Fernsehen übertragen und hat entsprechend eine größere Reichweite zu einem räumlich weit entfernten Publikum. Hier zeigt sich, dass unter dem Einfluss der neuen religiösen Marktbedingungen, die den Denominationalismus seit einigen Jahrzehnten zunehmend überlagern, Gemeinden immer stärker zu relativ unabhängigen ,organisationalen“ Akteuren werden, die um ein lokales religiöses Publikum konkurrieren, das sie fortlaufend überzeugen müssen (Kern und Pruisken 2018). Die Zugangsbarrieren für die Teilnahme sinken dabei ab: Prinzipiell kann jede Interessierte teilnehmen, wodurch es Mittel der symbolischen Reproduktion und professionalisierter Inszenierungen bedarf, um das relativ fragmentierte und fluide Publikum (zumindest situativ) einzubinden.

Verhältnis von Mitteln der symbolischen Reproduktion (4), Inszenierung (5), Darstellern (2) und Publikum (3) In vielen südlichen US-Metropolen ist eine eigene Infrastruktur entstanden, die die Megakirchen bei der Inszenierung religiöser Events unterstützt. Es braucht professionelle Musiker; die Licht- und Tontechnik muss auf die spezifischen Erfordernisse von Gottesdiensten spezialisiert sein. Zudem benötigt die Gemeinde ein erfahrenes, theologisch mindestens rudimentär geschultes und zugleich technisch versiertes Personal, das Gottesdienste inszenieren kann. Neben einem großen und professionalisierten Mitarbeiterstamm verfügt die Lakewood Church über eine Worship-Band, die eigene christliche Musik produziert ${ }^{11}$, und unterscheidet sich dahingehend von kleineren Megakirchen, die oft nur die Musik der erfolgreichen christlichen Pop-Bands covern und deshalb auch nicht live streamen dürfen.

Der wichtigste sichtbare Unterschied im Gottesdienst der Megakirche aus dem Jahr 2019, ${ }^{12}$ der per Video online verfügbar ist, im Unterschied zu dem zuvor besprochenen Gottesdienst sind die Mittel der symbolischen Reproduktion, die zur Verfügung stehen. Die Lakewood Church veranstaltet ihre Gottesdienste in einem umgebauten ehemaligen Basketballstadion, das sie 2005 gekauft hat und das 16.000 Zuschauerinnen fasst. Der Bühnen- und Zuschauerraum ähnelt in keiner Weise einer „klassischen“ Kirche. Die Gemeinde verfügt über alle Möglichkeiten der moder-

\footnotetext{
$11 \mathrm{https}: / / \mathrm{www} .1$ lakewoodchurch.com/store/music.

12 https://www.lakewoodchurch.com/watch-online/2019/November/2019-11-17-1100--joelosteen.
} 
nen Licht- und Bühnentechnik (schwenkende Kamera, Beleuchtung, professionelle Medientechnik). Der Zuschauerraum ist bestuhlt. Lichttechnik, Musik und Predigt folgen einem ausgefeilten Konzept: „Lakewood Church's worship leaders (i.e. musicians) and the light ministry team consciously aim for a certain worship service atmosphere - joyous and celebratory during the praise phase, more toned down and intimate during the worship phase, and full of brightness during Osteen's prayer and message of hope" (Rakow 2020, S. 102). Rakow zeigt in ihrer Studie detailliert, wie Licht und Musik gezielt für die Erzeugung einer fokussierten Atmosphäre eingesetzt werden.

Indem der Gottesdienst sich einerseits an den Standards moderner Popmusik und Shows orientiert sowie andererseits zentrale Leitideen eines ,therapeutischen Diskurses“ aufgreift und religiöse Inhalte in den Hintergrund drängt, werden kulturelle Extension und psychologische Identifikation für ein breites Publikum möglich. Die Gemeinde verfolgt eine Universalisierungsstrategie, bei der die Distanzen zwischen verschiedenen ethnischen, politischen und ideologisch gebundenen Gruppen mit popkulturellen Elementen überbrückt werden. Innerhalb der USA ist die Gemeinde mit dieser Strategie erfolgreich, im säkularen Europa konnten die Megakirchen kein Wachstum in solchem Ausmaß verzeichnen.

Authentizität wird in diesem Fall also in der Inszenierung erzeugt. Sie beruht nicht, wie im ersten Fall, auf direkten persönlichen und religiösen Bindungen. Die Anwesenden kennen sich persönlich nicht, und sie sind häufig auch nur in geringem Maße mit religiösem Wissen vertraut. Obwohl die Lakewood Church das Fernsehen und die digitalen Plattformen nutzt und der Gottesdienst gezielt auch an das nicht anwesende Publikum gerichtet ist, lebt die Inszenierung von Authentizität in diesem Fall noch von der körperlichen Kopräsenz der Zuschauerinnen. Die Darsteller spielen für das anwesende Publikum. Die Kamera fährt gezielt an besonders aktive oder emotionale Zuschauer heran, um das nicht anwesende Publikum teilhaben zu lassen. Wie für Events typisch, entfaltet der Gottesdienst seine Wirkung, weil er durch die aufwändige Inszenierung zu einer außeralltäglichen Erfahrung wird. Das Aufgehen des Einzelnen im „ganz Anderen“ und das Teilen dieser Grenzüberschreitung mit fremden Personen wird hier als besonders befreiend und authentisch erlebt (Gebhardt 2008, S. 207-209). Der Erfolg der Lakewood Church, die jeden Sonntag etwa 40.000 anwesende Besucherinnen anzieht, stützt diese These.

\subsection{Fall 3: Der Youtube-Vlog als religiöse Performance: ,kirby is a boss“" Verhältnis des Systems der kollektiven Repräsentation (1) zu den Akteuren (2)}

Die YouTuberin Kirby Kelly spricht im Rahmen ihrer Videos nicht als Vertreterin einer Denomination oder Gemeinde und damit als Trägerin einer bestimmten Rolle, sondern tritt als Privatperson auf. Zwar bewegt sie sich vor dem Hintergrund eines evangelikalen Glaubensverständnisses, dies wird den Zuschauerinnen aber nicht unbedingt unmittelbar klar. Dementsprechend ist sie frei bei der Interpretation des Skriptes und kann es sich zu eigen machen. Das Video beinhaltet im untersuchten Fall eine Nacherzählung ihres Konversionserlebnisses. Kelly nimmt in ihrem Video auf die bewusste Entscheidung zum christlichen Glauben Bezug, die den Bruch mit dem sündigen Leben und die Hinwendung zu einer christlichen 
Lebensführung markiert und damit eine übergeordnete Rolle in jeder evangelikalen Biographie spielt (Kern 1997). In anderen Videos berichtet sie aus ihrem Alltag oder lässt ihre Followers an wichtigen Lebensereignissen teilhaben. Das Publikum kann daher kaum zwischen dem ,echten“ Leben der Darstellerin und der Inszenierung unterscheiden. Im Rahmen der Erzählung kommt es zu einer Verschmelzung von Darstellerin und Skript. Das Publikum wird im Zuge des Videos durch Krisen und Wendungen, die Ausbreitung des Privatlebens, den Einsatz emotionaler, sich steigernder oder abschwellender Musik sowie durch Tränenausbrüche oder Ausdrücke des Glücks durchgehend emotional angesprochen und zur Identifikation angeregt. Die Darstellerin investiert ,echte“ Emotionen in die Darstellung und erlebt ihre religiöse Erfahrung vor der Kamera quasi noch einmal. Es ist für das Publikum nicht $\mathrm{zu}$ erkennen, ob es sich um eine Inszenierung handelt oder nicht.

In einfachen Interaktionssystemen - dazu zählen Gottesdienste, seelsorgerischer Gespräche und Events - konstituiert sich das Publikum über körperliche Anwesenheit (Luhmann 1972; Collins 2014). Im Zuge der Digitalisierung ist Anwesenheit allerdings zunehmend virtuell möglich, so dass das Publikum räumlich und zeitlich differenziert und in diesem Sinne noch individualisierter und fragmentierter wird (Webster und Ksiazek 2012). So sind auch die Zuschauerinnen der Influencerin sowohl räumlich und zeitlich als auch untereinander stark fragmentiert. Die Videos der Darstellerin lassen sich auch Jahre später und von anderen Kontinenten aus ansehen, so dass zentrale Elemente der Performance (Darsteller und Publikum) potenziell einen maximalen Grad an raumzeitlicher Erreichbarkeit aufweisen. So kann theoretisch nicht vorausgesetzt werden, dass das Publikum über den gleichen kulturellen Hintergrund und Bezugsrahmen wie die Darstellerin verfügt. Allerdings ist es sehr wahrscheinlich, dass ihre Videos von Algorithmen gefiltert und dementsprechend im weitesten Sinne ihrer Zielgruppe - junge, primär evangelikale Frauen - vorgeschlagen werden. Sie bewegt sich zwar mit ihren Videoblogs in den Genregrenzen säkularer Lifestyle-Videos und versucht, über die Grenzen des evangelikalen Feldes hinaus möglichst viele User anzusprechen, grenzt sich allerdings eindeutig durch christliche Codes wie „,devil“ oder „Jesus“ von der säkularen Welt ab.

Verhältnis von Mitteln der symbolischen Reproduktion (4), Inszenierung (5), Akteuren (2) und Publikum (3) Mit den Plattform-Interfaces werden in vielerlei Hinsicht neue Mittel der symbolischen Reproduktion mitgeliefert, allerdings nicht nur durch die Plattformen, sondern auch durch die Ausbreitung nuancierter Emoticons, Kamera-Filter oder LED-Technologien auf der Ebene von Smartphones. Die Social-Media-Influencerin nutzt für ihre Darstellungen in diesem Sinne Mittel der symbolischen Reproduktion, die erst im Kontext der Plattform möglich werden. So wird Kirby - trotz des betont legeren Settings - perfekt ausgeleuchtet, während die einzelnen Sequenzen der Erzählung durch professionelle Schnitttechniken nicht ausufern, sondern unmittelbar aneinander anknüpfen und stimulierende Brüche erzeugen. Auf diese Weise ist sie in der Lage, eine professionelle Inszenierung zu produzieren, die eine große Reichweite erzielt, ohne dass sie über eine eigene Organisationsstruktur verfügen muss.

Zudem nutzt sie performative Mittel wie den Aufbau eines abgeschlossenes Narratives, zahlreiche Wiederholungen ihrer zentralen Message sowie kognitive Ver- 
einfachungen, wobei die räumliche Distanz zwischen der Darstellerin und ihrem Publikum in erster Linie durch die Inszenierung von Nähe und einer privaten, alltäglichen Atmosphäre überbrückt wird. So sitzt Kirby Kelly im Rahmen des Videos in betont legerer Kleidung auf ihrem Bett und lässt, auch durch die Notizzettel und Fotos im Hintergrund, einen Einblick in ihre Privatsphäre zu. Sie heißt dabei das Publikum in ,ihrem Zimmer“ willkommen und spielt die Distanz zwischen sich und den Zuschauern mit Formulierungen herunter wie: „Hey guys ...“ oder „You guys know me" oder "love you“. Auf diese Weise wird eine hohe kulturelle Extension erzeugt. Trotz eindeutig christlicher Markierungen und einer Strategie der Partikularisierung werden die Inhalte voraussetzungsarm und verständlich kommuniziert.

Betonungen persönlicher Krisen - wie Mobbing, Depression und dem Tod des Vaters sowie von Krisen der Familie im Kontext von 9/11 - stellen Identifikationsmöglichkeiten für ein breites Publikum bereit. Die gelungene psychologische Identifikation des Publikums zeigt sich in den Kommentaren unter dem hier untersuchten Video. So heißen sich Zuschauer gegenseitig in der "Community“ oder „Family“ willkommen und kommentieren wiederholt, dass sie das Video zum Weinen oder sogar zum Konvertieren gebracht habe. Im Gegensatz zu religiösen Veranstaltungen vor Ort oder öffentlichen Live-Übertragungen werden dem Publikum von der Influencerin allerdings kaum Vernetzungs- oder Begegnungsmöglichkeiten angeboten. Sie sind im Falle von YouTube ausschließlich auf die Kommentarfunktion unter dem jeweiligen Video beschränkt, wo Zuschauerinnen das Video kommentieren und die Kommentare der anderen lesen, ,liken“ oder beantworten können. Dies kompensiert die Influencerin jedoch über eine andauernde und teilweise auch Live-Präsenz ${ }^{13}$ auf weiteren Plattformen wie Instagram, TikTok oder Online-Jugendkonferenzen. ${ }^{14}$ Dort können die Zuschauerinnen einen tieferen Einblick in das Leben von Kirby Kelly erhalten, mit ihr und untereinander interagieren und eine Bindung aufbauen.

Authentizität wird hier durch das Verschmelzen der Darstellerin mit ihrer Rolle und dem Skript erzeugt. Das Publikum - mit seiner Körperlichkeit und seinen Emotionen - ist räumlich und zeitlich fragmentiert und körperlich nicht anwesend. Der Erfolg der Performance zeigt sich zwar in den hohen Klickzahlen sowie den Kommentaren in den Kommentarspalten. Dem Publikum wird insofern ermöglicht, sich als Teil eines Kollektivs zu fühlen. Die religiöse Erfahrung wird aber durch das Video selbst vermittelt und speist sich weniger aus der kollektiven Erfahrung.

\footnotetext{
13 https://www.instagram.com/tv/CGNzls81XUQ/.

14 https://www.howtolifemovement.com/the-z-conference.
} 


\section{Schluss}

Die Popularisierung der Religion lässt sich vor diesem Hintergrund theoretisch als Begleiterscheinung von Prozessen der sozialen ,Ebenendifferenzierung “15 interpretieren: Die Makro-, Meso- und Mikroebenen der Religion (Kultur, gesellschaftliche Institutionen und Organisationen, Personen) entwickeln sich auseinander und werden voneinander unabhängiger (Luckmann 1988). Anders formuliert: Die Teilnahme am Gottesdienst ergibt sich seltener aus lokalen oder sozialen Zugehörigkeiten. Vielmehr finden sich im religiösen Ritual häufig Menschen zusammen, die sich einerseits nicht kennen und andererseits über unterschiedliches kulturelles Hintergrundwissen verfügen. In digitalisierten Ritualen fehlt zudem die räumliche Kopräsenz. Die These des Beitrags war, dass die Anforderungen an die Inszenierung steigen, wenn die Distanzen zwischen kulturellem Hintergrundwissen, Darstellern und Publikum im Ritual zunehmen. Die Performance kann nur gelingen, wenn ihre einzelnen Komponenten re-fusioniert werden: Erst wenn das Publikum sich eins fühlt mit der Darstellerin und dabei vergisst, dass es sich „nur“ um eine Inszenierung handelt, wirkt die Performance ,authentisch“.

Allerdings müssen nicht alle Ritualkomponenten gleichermaßen re-fusioniert werden. Vielmehr kommt es auf den sozialen, organisatorischen und institutionellen Kontext an, indem sich das Ritual vollzieht. Authentizität wird in allen drei hier untersuchten Fällen erzeugt, aber auf unterschiedliche Weise:

Im Gottesdienst der traditionellen evangelikalen Gemeinde sind die Elemente einer Performance vergleichsweise wenig differenziert. Viele Gemeindemitglieder beteiligen sich aktiv am Gottesdienst. Darsteller und Publikum sind daher oft identisch. Das Skript weicht nur in geringem Maße vom baptistischen Hintergrund ab. Die Inszenierung gelingt, weil sie sich an etablierte Mitglieder der Gemeinde richtet, die mit dem religiösen Hintergrund und dem Ablauf vertraut sind und sich darüber hinaus persönlich kennen. Religiöse Erfahrung, Vergemeinschaftungserfahrung und kultureller Hintergrund fallen vergleichsweise stark zusammen.

Der Gottesdienst der marktorientierten Megakirche „Lakewood Church“ zeichnet sich durch ein hohes Maß an sozialer Differenzierung aus. Als ressourcenstarke Organisation mit einer ausdifferenzierten, professionalisierten Rollenstruktur verfügt die Gemeinde über die erforderlichen Mittel der symbolischen Reproduktion, um die Distanz zwischen einem heterogenen Publikum und den Darstellern erfolgreich zu überbrücken. Die Gemeinde nutzt popkulturelle Elemente wie das positive Denken und Anekdoten aus dem Alltag, um die breite Masse anzusprechen, sowie Licht- und Musikeffekte, um Emotionen zu erzeugen. Der Live-Charakter sowie die rhythmische Steigerung der Inszenierung mit Hilfe von Musik und Lichteffekten ermöglichen es dem heterogenen sowie religiös individualisierten Publikum, sich mitreißen zu lassen. Authentizität entsteht hier in der Performance, weil Publikum,

\footnotetext{
15 Das Konzept der Ebenendifferenzierung wurde in der deutschsprachigen Soziologie vor allem von Religionssoziologen aufgegriffen (Pollack 2016; Pollack und Rosta 2015; Kern und Pruisken 2017; Petzke 2013), weil insbesondere Luhmann die Problematik der Ebenendifferenzierung selbst maßgeblich am Beispiel der Religion ausbuchstabiert hat. Wir folgen in diesem Aufsatz nur lose an Luhmanns Vorstellung der Ebenendifferenzierung und orientieren uns an einer handlungstheoretischen Interpretation, siehe Pollack (2016), Luckmann (1988) oder Schimank (2005).
} 
Darsteller und religiöser Hintergrund in der Inszenierung verschmelzen. Der Pastor präsentiert sich der Öffentlichkeit wie ein „Prominenter“ im klassischen Sinne als ,intimate stranger“ (Schickel 2000). Gerade die distanzierte Beziehung zur Gemeinde ermöglicht die Überhöhung des Darstellers (Jerslev 2016, S. 5238) und die Zuschreibung von Heiligkeitsqualitäten. Nach jedem Gottesdienst besteht die Möglichkeit, Joel Osteen persönlich zu treffen, ihm die Hand zu schütteln und ein Foto mit ihm zu machen.

Der Youtube-Vlog einer erfolgreichen „Christfluencerin“ überbrückt noch größere räumliche und zeitliche Distanzen. Mit der Entstehung digitaler Plattformen werden die Ebenen der individuellen religiösen Erfahrung und der Organisation ein weiteres Mal unabhängiger, weil die Plattformorganisationen eine „evaluative Infrastruktur“ bereitstellen, die räumliche und zeitliche Begrenzungen auflöst und religiöse Akteure von dem Ressourcenaufwand, den die Megakirchen noch betreiben müssen, befreit. Anders als Osteen, der in seiner Rolle als Pastor auftritt, hebt die Influencerin die Trennung zwischen ihrer Person und dem Skript auf, indem sie ihre eigene Konversionserfahrung noch einmal aufführt. Auf diese Weise werden Skript und Darstellerin miteinander verschmolzen. Durch die Inszenierung von Nähe und Intimität wird gezielt eine Verbindung zum Publikum erzeugt. Die langjährige Präsenz der Darstellerin auf einer Vielzahl von Online-Plattformen ermöglicht zudem die fortwährende Teilhabe von Zuschauern bzw. „Followers“ an der Entwicklung ihres Lebens im Sinne eines umfangreichen, sich stetig entwickelnden Narratives (Ostertag 2021). Über die Zeit wird ein gemeinsames Hintergrundwissen etabliert und Vertrauen aufgebaut.

Die Popularisierung der Religion beinhaltet insofern einen aktiven Ablösungsund Wiederaneignungsprozess. Die religiösen Akteure - individuell wie organisational - distanzieren sich zunächst aktiv von der ,starken“, expertenorientierten Hintergrundkultur der religiösen Wertsphäre und (re-)produzieren aus dieser Distanzierung heraus die populäre Religion. Dieser Prozess vollzieht sich im Rahmen der Entstehung organisatorischer Infrastrukturen wie Plattformen oder marktorientierter Gemeinden. Sie stellen die organisatorischen und institutionellen Bedingungen bereit, die die Performance ermöglichen.

Auch kleine Gemeinden nutzen natürlich Performances. Denn auch der Gottesdienst der kleineren baptistischen Gemeinde ist eine Inszenierung - hier werden ebenfalls Darstellerinnen, Licht, Ton etc. in „Szene“ gesetzt. Dieser Gottesdienst wird allerdings für ein bekanntes, mit der Gemeinde verbundenes Publikum inszeniert, das mit den kulturellen Konventionen des Baptismus vertraut ist. Die Popularisierung (oder auch Emotionalisierung) der Religion ist in diesem Fall weniger notwendig. Sie wird es erst dann, wenn ein räumlich, zeitlich und kulturell fragmentiertes Publikum erreicht werden soll.

Funding Open Access funding enabled and organized by Projekt DEAL.

Open Access Dieser Artikel wird unter der Creative Commons Namensnennung 4.0 International Lizenz veröffentlicht, welche die Nutzung, Vervielfältigung, Bearbeitung, Verbreitung und Wiedergabe in jeglichem Medium und Format erlaubt, sofern Sie den/die ursprünglichen Autor(en) und die Quelle ordnungsgemäß nennen, einen Link zur Creative Commons Lizenz beifügen und angeben, ob Änderungen vorgenommen wurden. 
Die in diesem Artikel enthaltenen Bilder und sonstiges Drittmaterial unterliegen ebenfalls der genannten Creative Commons Lizenz, sofern sich aus der Abbildungslegende nichts anderes ergibt. Sofern das betreffende Material nicht unter der genannten Creative Commons Lizenz steht und die betreffende Handlung nicht nach gesetzlichen Vorschriften erlaubt ist, ist für die oben aufgeführten Weiterverwendungen des Materials die Einwilligung des jeweiligen Rechteinhabers einzuholen.

Weitere Details zur Lizenz entnehmen Sie bitte der Lizenzinformation auf http://creativecommons.org/ licenses/by/4.0/deed.de.

\section{Literatur}

Alexander, Jeffrey C. 2004. Cultural pragmatics: social performance between ritual and strategy. Sociological Theory 22:527-573.

Ammerman, Nancy T. 2016. Denominations, congregations, and special purpose groups. In Handbook of religion and society. Handbooks of sociology and social research, Hrsg. David Yamane, 133-154. Cham: Springer.

von Appen, Ralf. 2013. Schein oder Nicht-Schein? Zur Inszenierung von Authentizität auf der Bühne. In Ware Inszenierungen Beiträge zur Popularmusikforschung, Bd. 39, Hrsg. Dietrich Helms, Thomas Phleps, 41-69. Bielefeld: transcript.

Balik, Shelby M. 2014. Rally the scattered believers. Northern new england's religious geography. Bloomington: Indiana University Press.

Beckert, Jens. 2009. The social order of markets. Theory and Society 38:245-269.

Berger, Peter L. 1963. A market model for the analysis of ecumenicity. Social Research 30:77-93.

Berger, Peter L. (Hrsg.). 2005. The desecularization of the world. Resurgent religion and world politics, 6. Aufl., Washington, DC: Ethics and Public Policy Center.

Berlinerblau, Jacques. 2001. Toward a sociology of heresy, orthodoxy, and doxa. History of Religions 40:327-351.

Bourdieu, Pierre. 1993. Die feinen Unterschiede. Kritik der gesellschaftlichen Urteilskraft. Frankfurt: Suhrkamp.

Bowler, Kate. 2013. Blessed. A history of the American prosperity gospel. New York: Oxford University Press.

Callon, Michel, und Fabian Muniesa. 2005. Economic markets as calculative collective devices. Organization Studies 26:1229-1250.

Chaves, Mark. 2006. All creatures great and small: Megachurches in context. Review of Religious Research 47:329-346.

Chaves, Mark, Shawna L. Anderson, und Alison Eagle. 2014. National congregations study. Cumulative data file and codebook. Durham: Duke University, Department of Sociology.

Collins, Randall. 2014. Interaction Ritual Chains. Princeton: Princeton University Press.

van Dijck, José. 2013. Facebook and the engineering of connectivity. A multi-layered approach to social media platforms. Convergence: The International Journal of Research into New Media Technologies 19:141-155.

DiMaggio, Paul J. 1987. Classification in Art. American Sociological Review 52:440-455.

Ebertz, Michael N. 2000. Transzendenz im Augenblick. Über die „Eventisierung“ des Religiösen - dargestellt am Beispiel der Katholischen Weltjugendtage. In Events. Soziologie des Außergewöhnlichen. Erlebniswelten, Bd. 2, Hrsg. Winfried Gebhardt, Ronald Hitzler, und Michaela Pfadenhauer, 345-362. Wiesbaden: VS.

Einspänner-Pflock, Jessica, und Werner Reichmann. 2014. „Digitale Sozialität“ und die „,synthetische Situation“. Konzeptionen mediatisierter Interaktion. In Die Mediatisierung sozialer Welten. Synergien empirischer Forschung. Medien $\bullet$ Kultur $\bullet$ Kommunikation, Hrsg. Friedrich Krotz, Cathrin Despotović, und Merle-Marie Kruse, 53-72. Wiesbaden: Springer VS.

Finke, Roger, und Rodney Stark. 1988. Religious economies and sacred canopies: religious mobilization in American cities, 1906. American Sociological Review 53:41-49.

Fischer-Lichte, Erika, und Isabel Pflug (Hrsg.). 2000. Inszenierung von Authentizität. Theatralität, Bd. 1. Tübingen: Francke.

Fligstein, Neil, und Luke Dauter. 2007. The sociology of markets. Annual Review of Sociology 33:105-128.

Forschungskonsortium, W.J.T. 2007. Megaparty Glaubensfest. Weltjugendtag: Erlebnis - Medien - Organisation. Bd. 12. Wiesbaden: VS. 
Funk, Wolfgang, und Lucia Krämer (Hrsg.). 2011. Fiktionen von Wirklichkeit. Authentizität zwischen Materialität und Konstruktion. Bielefeld: transcript.

Gebhardt, Winfried. 2000. Feste, Feiern und Events. Zur Soziologie des Außergewöhnlichen. In Events. Soziologie des Außergewöhnlichen. Erlebniswelten, Bd. 2, Hrsg. Winfried Gebhardt, Ronald Hitzler, und Michaela Pfadenhauer, 17-31. Wiesbaden: VS.

Gebhardt, Winfried. 2008. Gemeinschaften ohne Gemeinschaft. Über situative Event-Vergemeinschaftungen. In Posttraditionale Gemeinschaften. Theoretische und ethnografische Erkundungen. Erlebniswelten, Bd. 14, Hrsg. Ronald Hitzler, Anna Honer, und Michaela Pfadenhauer, 202-213. Wiesbaden: VS.

Gebhardt, Winfried. 2015. Feste, Feiern und Events. Die etwas andere Freizeit. In Handbuch Freizeitsoziologie, Hrsg. Renate Freericks, Dieter Brinkmann, 415-429. Wiesbaden: Springer.

Gebhardt, Winfried. 2018. Religiöse Szenen und Events. In Handbuch Religionssoziologie. Veröffentlichungen der Sektion Religiosoziologie der Deutsche Gesellschaft für Solziologie, Hrsg. Detlef Pollack, Volkhard Krech, Olaf Müller, und Markus Hero, 591-610. Wiesbaden: Springer VS.

Gebhardt, Winfried, Ronald Hitzler, und Michaela Pfadenhauer (Hrsg.). 2000. Events. Soziologie des Außergewöhnlichen. Opladen: Leske \& Budrich.

Gerhards, Jürgen. 2001. Der Aufstand des Publikums. Eine systemtheoretische Interpretation des Kulturwandels in Deutschland zwischen 1960 und 1989. Zeitschrift für Soziologie 30:163-184.

Geser, Hans. 1999. Zwischen Anpassung, Sebstbehauptung und politischer Agitation. Zur aktuellen (und zukünftigen) Bedeutung religiöser Organisationen. In Institution, Organisation, Bewegung: Sozialformen der Religion im Wandel, Hrsg. Michael Krüggeler, Karl Gabriel, und Winfried Gebhardt, 39-69. Opladen: Leske + Budrich.

Gillespie, Tarleton. 2010. The politics of ,platforms“. New Media \& Society 12:347-364.

Goffman, Erving. 1973. Interaktion. Spaß am Spiel, Rollendistanz. München: Piper.

Graf, Friedrich Wilhelm. 2007. Die Wiederkehr der Götter. Religion in der modernen Kultur, 1. Aufl., Bd. 1779. München: Beck.

Gramsci, Antonio. 1985. Selections from the prison notebooks. New York: International Publishers.

Haken, Meike, und Michael Wetzels. 2017. Jesus Christ Football Star? Hybride Gottesdienste im Spannungsfeld intendierter Liturgie und Publikumspartizipation. In Hybride Events, Hrsg. Gregor J. Betz, Ronald Hitzler, Arne Niederbacher, und Lisa Schäfer, 201-215. Wiesbaden: Springer.

Hans, Barbara. 2017. Inszenierung von Politik. Zur Funktion von Privatheit, Authentizität, Personalisierung und Vertrauen. Wiesbaden: VS.

Heiser, Patrick, Christian Kurrat, und Christian Ludwig (Hrsg.). 2014. Sozialformen der Religionen im Wandel. Wiesbaden: Springer VS.

Henning, Christoph. 2015. Theorien der Entfremdung. zur Einführung. Hamburg: Junius.

Hepp, Andreas, Veronika Krönert, und Waldemar Vogelgesang. 2009. Mediatisierte Religion: Die Mediatisierung des Religiösen am Beispiel des XX. Weltjugendtags. In Theatralisierung der Gesellschaft Medientheatralität und Medientheatralisierung, Bd. 2, Hrsg. Herbert Willems, 117-136. Wiesbaden: VS.

Herberg, Will. 1960. Protestant, catholic, jew: an essay in American religious sociology. New York: Knopf Doubleday.

Hjarvard, Stig. 2013. The mediatization of society and culture. London: Routledge.

Hracs, Brian J., und Jack Webster. 2020. From selling songs to engineering experiences. Exploring the Competitive Strategies of Music Streaming Platforms. Journal of Cultural Economy 14:240-257.

Iannaccone, Laurence R. 1992. Religious markets and the economics of religion. Social Compass 39:123-131.

Jerslev, Anne. 2016. In the time of the microcelebrity. Celebrification and the youtuber Zoella. International Journal of Communication 10:5233-5251.

Kern, Thomas. 1997. Zeichen und Wunder. Enthusiastische Glaubensformen in der modernen Gesellschaft. Frankfurt am Main, Berlin, Bern, New York, Paris, Wien: Peter Lang.

Kern, Thomas. 2017. Jeffrey Alexander und die Kultursoziologie. In Begriffe - Kontexte - Perspektiven - Autor_innen Handbuch Kultursoziologie, Bd. 1, Hrsg. Stephan Moebius, Frithjof Nungesser, und Katharina Scherke, 1-8. Wiesbaden: Springer.

Kern, Thomas, und Insa Pruisken. 2017. Kontingenzbewältigung durch Organisation. In Religion soziologisch denken. Reflexionen auf aktuelle Entwicklungen in Theorie und Empirie. Veröffentlichungen der Sektion Religionssoziologie der Deutschen Gesellschaft für Soziologie, Hrsg. Heidemarie Winkel und Kornelia Sammet, 407-427. Wiesbaden: Springer Fachmedien Wiesbaden.

Kern, Thomas, und Insa Pruisken. 2018. Was ist ein religiöser Markt? Zum Wandel der religiösen Konkurrenz in den USA. Zeitschrift für Soziologie 47:29-45. 
Kern, Thomas, und Insa Pruisken. 2020. Der Wandel religiöser Inklusion in den USA: Von der ,traditionellen“ zur ,,vernetzten“ Religiosität? In Die Refiguration der Religion. Perspektiven der Religionssoziologie und Religionswissenschaft, Hrsg. Hubert Knoblauch, 147-168. Weinheim: Beltz Juventa.

Kern, Thomas, und Uwe Schimank. 2013. Megakirchen als religiöse Organisationen: Ein dritter Gemeindetyp jenseits von Kirche und Sekte? Kölner Zeitschrift für Soziologie und Sozialpsychologie 65:285-309.

Knoblauch, Hubert. 2000a. Das strategische Ritual der kollektiven Einsamkeit. Zur Begrifflichkeit und Theorie des Events. In Events. Soziologie des Außergewöhnlichen. Erlebniswelten, Bd. 2, Hrsg. Winfried Gebhardt, Ronald Hitzler, und Michaela Pfadenhauer, 33-50. Wiesbaden: VS.

Knoblauch, Hubert. 2000b. Das strategische Ritual der kollektiven Einsamkeit. Zur Begriffiichkeit und Theorie des Events. In Events. Soziologie des Außergewöhnlichen, Hrsg. Winfried Gebhardt, Ronald Hitzler, und Michaela Pfadenhauer, 33-50. Opladen: Leske \& Budrich.

Knoblauch, Hubert. 2002. Ganzheitliche Bewegungen, Transzendenzerfahrung und die Entdifferenzierung von Kultur und Religion in Europa. Berliner Journal für Soziologie 12:295-307.

Knoblauch, Hubert. 2009. Populäre Religion. Auf dem Weg in eine spirituelle Gesellschaft. Frankfurt am Main: Campus.

Knoblauch, Hubert. 2018. Jenseits von Orthodoxie und Heterodoxie: Das populäre Wissen. In Heterodoxie. Konzepte, Traditionen, Figuren der Abweichung, Hrsg. Michael Schetsche, Ina Schmied-Knittel. Köln: Herbert von Halem Verlag.

Knoblauch, Hubert (Hrsg.). 2020. Die Refiguration der Religion. Perspektiven der Religionssoziologie und Religionswissenschaft. Weinheim: Beltz Juventa.

Kornberger, Martin, Dane Pflueger, und Jan Mouritsen. 2017. Evaluative infrastructures: accounting for platform organization. Accounting, Organizations and Society 60:79-95.

Krech, Volkhard, Jens Schlamelcher, und Markus Hero. 2013. Typen religiöser Sozialformen und ihre Bedeutung für die Analyse religiösen Wandels in Deutschland. Kölner Zeitschrift für Soziologie und Sozialpsychologie 65:51-71.

Kreuzer, Stefanie. 2011. Künstl(er)i(s)che Strategien von Authentizitätskonstruktion. Beispiele aus Literatur, Film und bildender Kunst. In Fiktionen von Wirklichkeit. Authentizität zwischen Materialität und Konstruktion, Hrsg. Wolfgang Funk, Lucia Krämer, 179-204. Bielefeld: transcript.

Krücken, Georg, und Frank Meier. 2006. Turning the university into an organizational actor. In Globalization and organization. World society and organizational change, Hrsg. Gili S. Drori, John W. Meyer, und Hokyu Hwang, 241-312. Oxford: Oxford University Press.

Krüggeler, Michael, Karl Gabriel, und Winfried Gebhardt (Hrsg.). 1999. Institution, Organisation, Bewegung: Sozialformen der Religion im Wandel. Opladen: Leske + Budrich.

Luckmann, Thomas. 1967. The invisible religion: the problem of religion in modern society. London: MacMillan.

Luckmann, Thomas. 1988. Die massenkulturelle Sozialform der Religion. In Kultur und Alltag (Sonderband 6 der ,Sozialen Welt“), Hrsg. Hans-Georg Soeffner, 37-48. Göttingen: O. Schwartz.

Luhmann, Niklas. 1972a. Die Organisierbarkeit von Religion und Kirchen. In Religion im Umbruch, Hrsg. Jakob Wössner, 245-285. Stuttgart: Enke.

Luhmann, Niklas. 1972b. Einfache Sozialsysteme. Zeitschrift für Soziologie 1:51-65.

Mayring, Philipp. 2015. Qualitative Inhaltsanalyse. Grundlagen und Techniken, 12. Aufl., Weinheim: Beltz Verlagsgruppe.

Mead, Sidney E. 1954. Denominationalism: the shape of protestantism in America. Church History 23:291-320.

Miller, Vincent Jude. 2013. Consuming religion. Christian faith and practice in a consumer religion. New York: Continuum. Repr.

Moberg, Marcus, und Tuomas Martikainen. 2018. Religious change in market and consumer society: the current state of the field and new ways forward. Religion 48:418-435.

Molm, Linda D., Jessica L. Collett, und David R. Schaefer. 2007. Building solidarity through generalized exchange: a theory of reciprocity. American Journal of Sociology 113:205-242.

Moore, Robert Laurence. 1995. Selling God. American religion in the marketplace of culture. New York: Oxford University Press.

Niebuhr, H. Richard. 1957. The social sources of denominationalism. New York: Meridian Books.

Ostertag, Stephen F. 2021. A cultural sociology of social media. Social drama, cultural affordances and blogging in the wake of hurricane Katrina. Cultural Sociology 15:113-133.

Pace, Enzo. 2006. Salvation goods, the gift economy and charismatic concern. Social Compass 53:49-64.

Petzke, Martin. 2013. Weltbekehrungen. Zur Konstruktion globaler Religion im pfingstlich-evangelikalen Christentum. Bielefeld: transcript. 
Pfadenhauer, Michaela. 2008. Organisieren. Eine Fallstudie zum Erhandeln von Events. Wiesbaden: VS.

Pollack, Detlef. 2016. Modernisierungstheorie - revised: Entwurf einer Theorie moderner Gesellschaften. Zeitschrift für Soziologie 45:61.

Pollack, Detlef, und Gergely Rosta. 2015. Religion in der Moderne. Ein internationaler Vergleich. Frankfurt am Main, New York: Campus.

Possamai, Adam M. 2015. Popular and lived religions. Current Sociology 63:781-799.

Pruisken, Insa, Josefa Loebell, Nina Monowski und Thomas Kern. 2021. From Denominationalism to Market Standards: How Does the Religious Market Affect Authority Relations in Protestant Congregations? Research in the Social Scientific Study of Religion. Im Erscheinen.

Rakow, Katja. 2013. Therapeutic culture and religion in America. Religion Compass 7:485-497.

Rakow, Katja. 2020. The light of the world: mediating divine presence through light and sound in a contemporary megachurch. Material Religion 16:84-107.

Reichertz, Jo. 2001. Masken des Authentischen oder: Die Rückkehr des öffentlichen Menschen? Medien Praktisch: Medienpädagogische Zeitschrift Für die Praxis 4:16-25.

Roose, Jochen, Mike S. Schäfer, und Thomas Schmidt-Lux. 2017. Fans. Soziologische Perspektiven. Wiesbaden: Springer.

Sanders, George. 2015. Religious non-places. Corporate megachurches and their contributions to consumer capitalism. Critical Sociology 42:71-86.

Sandıkcı, Özlem. 2018. Religion and the marketplace: constructing the „new“ muslim consumer. Religion 48:453-473.

Schickel, Richard. 2000. Intimate strangers. The culture of celebrity in America. Chicago: Ivan R. Dee.

Schimank, Uwe. 2005. Differenzierung und Integration der modernen Gesellschaft. Beiträge zur akteurzentrierten Differenzierungstheorie 1. Wiesbaden: VS.

Simmel, Georg. 1908. Soziologie: Untersuchungen über die Formen der Vergesellschaftung. Leipzig: Duncker \& Humblot.

Stolz, Jörg. 2006. Salvation goods and religious markets: integrating rational choice and Weberian perspectives. Social Compass 53:13-32.

Suh, Daniel, und Raymond Russell. 2015. Non-affiliation, non-denominationalism, religious switching, and denominational switching: longitudinal analysis of the effects on religiosity. Review of Religious Research 57:25-41.

Taylor, Charles. 2003. The malaise of modernity. Concord: House of Anansi Press.

Turner, Victor. 1974. Liminal to liminoid, in play, flow, and ritual: an essay in comparative symbology. Rice University Studies 60:53-92.

Turner, Victor. 2005. Das Ritual. Struktur und Anti-Struktur. Frankfurt, New York: Campus.

Warner, R. Stephen. 1994. The place of the congregation in the contemporary American religious configuration. In American congregations: new perspectives in the study of congregations, Hrsg. James P. Wind, James W. Lewis, 54-99. Chicago: University of Chicago Press.

Weber, Max. 1980. Wirtschaft und Gesellschaft, 5. Aufl., Tübingen: Mohr.

Weber, Max. 1988. Gesammelte Aufsätze zur Religionssoziologie I. Tübingen: J.C.B. Mohr.

Webster, James G., und Thomas B. Ksiazek. 2012. The Dynamics of Audience Fragmentation: Public Attention in an Age of Digital Media. Journal of Communication 62:39-56. 